Investigaciones Fenomenológicas, n. 12, 2015, 33-50. e-ISSN: $1885-1088$

\title{
LAS RAÍCES EMPIRIOCRITICISTAS DEL CONCEPTO DE MUNDO NATURAL EN E. HUSSERL*
}

\author{
THE "EMPIRIOCRITICIST" SOURCES \\ OF the Concept of Natural WorLd In E. HUSSERL
}

\author{
Marco Cavallaro \\ University of Cologne, Alemania \\ cavallaro.marco@gmail.com
}

\begin{abstract}
Resumen: En este articulo queremos investigar la relación entre las filosofías de Edmund Husserl y Richard Avenarius. A pesar de que este tema ha sido prácticamente ignorado por los estudiosos de Husserl, es de suma importancia si uno quiere iluminar los orígenes del pensamiento fenomenológico de Husserl. Nuestra tesis es que el concepto de Husserl de actitud natural y su correlato, el mundo natural (Umwe/t) - tal y como ellos surgen en diferentes conferencias durante la década de 1910 y especialmente en Ideas I con respecto a la reducción fenomenológica- se derivan de una temprana confrontación con el punto de vista del empiriocriticismo de Avenarius como fue expuesto en su libro Der menschliche Weltbegriff. Con el fin de mantener esta afirmación, discutiremos el acercamiento gradual de Husserl a las teorías del filósofo suizo-alemán así como su última crítica al mismo. Aunque, de hecho, Husserl concibió el punto de vista de Avenarius como un principio valioso, al final de sus reflexiones pone en cuestión algunas de las premisas de la filosofía empiriocriticista.
\end{abstract}

Palabras clave: Edmundo Husserl | Richard Avenarius | Empiriocriticismol Mundo natural

\begin{abstract}
In this paper I investigate the relationship between the philosophies of Edmund Husserl and Richard Avenarius. Despite this topic has been almost disregarded by Husserlian scholars, it is of uttermost importance if one aims at illuminating the origins of Husserl's phenomenological thinking. My thesis is that Husserl's concept of natural attitude and its correlate, the natural world (Umwe/t) -as they emerge in various lectures during the 1910 s and especially in Ideen I with respect to the phenomenological reduction- stem from an early confrontation with Avenarius' "empiriocriticist" standpoint as laid out in his book Der menschliche Weltbegriff. In order to uphold this claim, I will discuss in my paper Husserl's gradual approach to the theories of the Swiss-German philosopher as well as his late critique of the same. Although Husserl in fact conceived of Avenarius' standpoint as a valuable beginning, he was decisively prompted in his late reflections to call into question some of the premises of the "empi rio-criticist" philosophy.
\end{abstract}

Key Words: Edmund Husserl I Richard Avenarius | Empiriocriticism | Natural World

*La traducción ha sido revisada por Axel Rivera Osorio and Sergio Pérez Gatica. 
Ich sehe darin eine sehr wertvolle Tendenz ${ }^{1}$

Edmund Husserl sobre Avenarius

\section{§ 1. ¿UN VIEJO DESIDERATUM DE LA FILOSOFÍA?}

En un famoso pasaje de Ser y Tiempo, Martin Heidegger llama la atención sobre un desiderátum fundamental que inquieta desde hace mucho tiempo a la filosofía, en cuyo cumplimiento, sin embargo, ésta fracasa una y otra vez." Este desiderátum consiste en "la elaboración de la idea de un 'concepto natural del mundo"' (Heidegger 2009, 60). Algunos años después de la publicación de la obra maestra de Heidegger, probablemente Edmund Husserl podría haberse sorprendido al encontrar una afirmación tal en la obra de su pupilo. Husserl, que en ese tiempo era ya Profesor Emérito de la universidad de Friburgo, de hecho la objetó en una conversación privada con Hans Reiner, la cual tuvo lugar el 31 de Diciembre de 1931, según relata Dorion Cairns. El problema consiste principalmente en que "Heidegger no menciona ni a Avenarius ni a Husserl cuando habla de la figura del mundo natural (natural world-picture), sino que la trata como si fuera una cuestión de conocimientos generales, en vez de un desarrollo de estos dos filósofos" (Cairns 1976, 63). Este comentario nos deja ver una discrepancia fundamental, aunque implícita, entre la consideración husserliana y heideggeriana del (sit venia verbo) 'pedigree' histórico-filosófico del concepto natural de mundo. Por una parte, Heidegger asume que ese concepto es relativamente antiguo, por eso es que puede decir que éste es el inexpresado desiderátum de la filosofía y que ni siquiera el pensamiento moderno ha podido realizar; asume que sólo una analítica existencial del Dasein, tal como la expone en la primera División de Ser y Tiempo, debería, eventualmente, llevar a cabo esta tarea. Por otro lado, el comentario lacónico que Husserl realiza sobre la distracción heideggeriana al no reconocer su deuda, en principio hacia su maestro e, indirectamente, hacia Avenarius, debería también sugerir el hecho de que Husserl considera al concepto de 'mundo natural' como un descubrimiento relativamente reciente de la filosofía contemporánea, y por ello, con una breve historia, cuyo origen debe ser rastreado en Avenarius y su desarrollo

\footnotetext{
1 "Veo en ello una tendencia muy valiosa".
} 
posterior en la fenomenología de Husserl.

La alta consideración que Husserl tenía por las obras del filósofo suizoalemán Richard Avenarius (1843-1896) perduró por una notable parte de su vida, desde las Investigaciones Lógicas (1900/01) hasta la Crisis (1936), es decir, por casi cuarenta años. Dentro de este periodo de tiempo, Husserl cambió más de una vez no sólo su valoración sino también su interpretación de la filosofía de Avenarius.

\section{$\S$ 2. La CRÍtica temprana de HuSSerl al PRINCIPIO de MaCh-Avenarius EN LOS PROLEGOMENA}

Sus oscilaciones en relación con el pensamiento del fundador del movimiento filosófico llamado 'Empirocriticismo' encuentra expresión ya desde el Capítulo noveno de los Prolegómenos para una lógica pura (1900). Aquí, Husserl toma en cuenta un tipo particular de 'fundación empírica de la lógica y de la teoría del conocimiento' semejante al psicologismo: en concreto "la fundamentación biológica de estas disciplinas, por medio del principio del menor esfuerzo, como lo llama Avenarius, o del principio de la economía del pensamiento, como lo llama Mach" (Husserl 2006, 165; Hua XVIII, 196). Aunque Husserl reconoce un cierto valor al Ilamado principio de Avenarius-Mach, especialmente en el contexto de una investigación psicológico-experimental, así como también en el desarrollo de un "doctrina técnica" (Kunstlehre) (Hua XVIII, § 54) de la lógica, sin embargo considera que "una ley psicológica o epistemológica, que habla de una tendencia [Bestreben] a lograr lo más posible en esto o aquello, es un absurdo [ein Unding]" (Husserl 2006, 174; Hua XVIII, 207). Una ley de este tipo, como cualquier intento de fundamentar la lógica pura sobre bases empíricas, eventualmente nos conduce a una petición de principio, en tanto que presupone la validez ideal del principio de la economía del pensamiento, una validez que ese principio excluye intrínsecamente (cf. Hua XVIII, § 56). Todavía, y a pesar de su vigorosa crítica, Husserl parece estar obligado, al menos parcialmente, a reconocer el mérito de la filosofía de Avenarius. De hecho, en una nota al principio del capítulo, Husserl declara: "La crítica adversa que he de llevar a cabo en este capítulo contra una tendencia capital de la filosofía de Avenarius, con- 
cuerda (verträgt sich) ${ }^{2}$ muy bien con la más alta estima por el investigador prematuramente arrebatado a la ciencia y por la solidez y seriedad de sus trabajos científicos" (Husserl 2006, 165; Hua XVIII, 196).

Si el pasaje antes citado de los Prolegomena deja ver una oscilación en las valoraciones de Husserl sobre el filósofo del empiriocriticismo Avenarius -la cual precisamente oscila entre una fuerte crítica de sus presuposiciones naturalistas y en un alto reconocimiento del valor de su proyecto filosófico general, una ambigüedad similar puede observarse también en lo que Husserl considera la interpretación del contenido de la filosofía de Avenarius. Efectivamente, después de haber expresado sus dudas sobre el punto de vista empirista de Avenarius en su obra temprana, Husserl define ese mismo punto de vista como una forma de "filosofía trascendental" (esto se encuentra en un largo parágrafo dedicado a la historia de la filosofía moderna desde Kant en La crisis) cuyo único defecto era representado por una falta de radicalismo, el cual subsecuentemente caracterizaría al proyecto fenomenológico y a su método (cf. Hua VI, 198). Más allá del hecho de que la noción husserliana de lo 'trascendental' no puede ser interpretada en términos del conocimiento histórico-filosófico común y que el propio Husserl puede ser difícilmente considerado como un confiable historiador de la filosofía, es per se notable la inclusión de Avenarius en la tradición de la filosofía-trascendental. De hecho, Husserl parece haber localizado en él como lo escribe ya en un manuscrito proveniente del curso de invierno de 1910/11, intitulado Problemas fundamentales de la fenomenología- "una tendencia muy valiosa (eine sehr wertvolle Tendenz)" (Hua XIII, 197). La naturaleza y los límites de esta 'valiosa tendencia' en la filosofía de Husserl es lo que debe ser evaluado en el presente artículo.

\section{§ 3. EXPERIENCIA PURA: EL PRINCIPIO DE INMANENCIA EN HUSSERL y AVENARIUS}

En primer lugar es bastante comprensible el hecho de que Husserl pensara encontrar en Avenarius, cuyo pensamiento es un tipo de filosofía positivista, una tendencia fructífera; especialmente si uno mira las presuposiciones que constituyen las bases metodológicas del trabajo de ambos pensadores. Como

\footnotetext{
${ }^{2}$ Nota del traductor. En la edición de Alianza (Husserl 2006, 165), el verbo alemán sich vertragen aparece traducido como "compadecerse", cuando debe ser traducido como "concordar" o "ser compatible", tal y como ha sido corregido ya en el cuerpo de este texto. 
Husserl lo expresa en un famoso pasaje de Ideas I: "Si 'Positivismo' quiere decir tanto como fundamentación, absolutamente exenta de prejuicios, de todas las ciencias en lo 'positivo', esto es, en lo que se puede captar originariamente, entonces somos NOSOTROS los auténticos positivistas. Nosotros, en efecto, no nos dejamos menoscabar por NINGUNA autoridad el derecho de reconocer todas las especies de intuición como fuentes de legitimidad del conocimiento igualmente valiosas - ni siquiera por la autoridad de la 'ciencia moderna de la naturaleza"' (Husserl 2013, 123; Hua III/1, 45). En este mismo sentido, Avenarius anuncia de forma programática en su voluminosa obra, la cual lleva por título Critica de la experiencia pura (1888, 2 tomos), su intento de llevar a cabo una crítica de la "experiencia pura", semejante a la de Kant, es decir, de la experiencia pre-científica y pre-filosófica. En el prefacio de dicha obra, Avenarius introduce su método de investigación casi con las mismas palabras que Husserl usará en su famoso llamado "a las cosas mismas", contenido en el prefacio del segundo volumen de las Investigaciones Lógicas: "en vez de volver a estos o aquellos filósofos, volver simplemente al punto natural de partida mismo y referirse -en vez de a libros- a las cosas" (Avenarius 1888, XXIII; cf. Hua XIX/1, 10 y Hua XXV, 21). ${ }^{3}$ El esfuerzo filosófico de Husserl y de Avenarius tiene en común la exclusión metódica o -para usar una expresión que cotejamos en ambos filósofos, aunque en contextos un poco diferentes (cf. Lavigne 2003, 2004) - la "puesta fuera de acción" (Ausschaltung) de los resultados de las teorías filosóficas y científicas como un paso fundamental para el descubrimiento del campo de la experiencia pura. Así, no es un misterio que la "filosofía inmanente", que es una expresión empleada recurrentemente por Husserl para caracterizar al Empirocriticismo de Avenarius, con su aversión hacia las presuposiciones teoréticas y además con su llamado a alcanzar un nivel de experiencia original y que no esté 'contaminado', Ilamara la atención de Husserl como un esfuerzo filosófico capaz de satisfacer los parámetros establecidos por el "principio de todos los principios (Prinzip aller Prinzipen)" (cf. Hua III/1, 51).

\section{§ 4. "El Concepto humano de Mundo"}

Si los presupuestos metódicos de Husserl y Avenarius no muestran diferencias insalvables - sino que más bien, como hemos visto, tienden a coincidir-,

\footnotetext{
3 "Statt auf diesen oder jenen Philosophen einfach auf den natürlichen Ausgangspunkt selbst 'zurückzugehen' und -statt an Bücher- unmittelbar an die Sachen 'anzuknüpfen'".
} 
la situación se vuelve mucho más complicada cuando se les pregunta a ambos filósofos la siguiente cuestión: ¿qué es lo que se da originariamente? Por paradójico que pueda parecer, una misma metodología no garantiza, en este caso, idénticos resultados. Pero discutamos una cosa a la vez.

La filosofía empiriocriticista defiende la tesis de que antes de que pueda surgir cualquier pensamiento teorético, nosotros vivimos ya en un mundo que tiene sus propias estructuras y sus rasgos distintivos. $Y$ no solamente eso, sino que el pensamiento teorético, tanto el científico como el filosófico, está basado en este mundo y encuentra además sus cimientos fundamentales en él. Este pensamiento está contenido en lo que Avenarius llama los dos "Axiomas empiriocríticos (empiriokritische Axiome)" en su crítica de la experiencia pura: 1) "Todos los contenidos de conocimiento de las visiones filosóficas del mundo [...] son modifcaciones de aquella asunción originaria [en concreto, la hipótesis de un mundo con sus estructuras peculiares]"4 ; 2) "todas las formas o medios especiales de conocimiento científico son desarrollos de formas precientíficas" ${ }^{5}$ (Avenarius 1888, VII; Cf. Avenarius 1891, Nr. 8, 5 s.). Si la filosofía y la ciencia deben su sentido y su justificación a la existencia de este mundo, entonces se hace evidente que este mundo debe ser investigado tal cual es dado en su forma originaria, es decir, debe realizarse una descripción del contenido de este mundo y esa es una tarea para cualquier proyecto que pretenda cumplir con el "principio de todos los principios", el cual nos exige que permanezcamos ante todo fieles a lo que es dado y en el modo en que nos es dado.

La tarea de describir el contenido del concepto originario de mundo no es expuesta por Avenarius en su obra principal, en la Crítica de la experiencia pura, sino en un texto, relativamente breve, intitulado El concepto humano de mundo (Der menschliche Weltbegriff), publicado en 1891. Aquí, el filósofo empiriocriticista ofrece la siguiente ilustración del concepto humano de mundo; así es como describe el mundo tal cual se encuentra en el inicios del filosofar: "Yo me encontraba con todos mis pensamientos y sentimientos en medio de un entorno [Umgebung]. Este entorno estaba compuesto de múltiples elementos, los cuales estaban entre sí en múltiples relaciones de dependencia. Al entorno per-

\footnotetext{
4 "Alle Erkenntnis-Inhalte der philosophischen Weltanschauungen [...] sind Abänderungen jener ursprünglichen Annahme".

5 "Alle speziellen wissenschaftlichen Erkenntnis-Formen oder -Mittel sind Ausbildungen vorwissenschaftlicher".
} 
tenecían también otros humanos con múltiples enunciaciones; y lo que decían estaba la mayoría de las veces nuevamente en una relación de dependencia con el entorno. [...] Todo, como yo mismo también: y así yo no pensaba otra cosa, que los otros humanos eran seres como yo - yo mismo un ser como ellos. Eso era el mundo, tal y como yo lo encontraba al inicio de mi filosofar, como algo existente, seguro, conocido, familiar, comprendido" ${ }^{6}$ (Avenarius 1891, Nr. 6 y 4). Es importante notar que la descripción que realiza Avenarius del concepto natural de mundo está llevada a cabo en pasado (en el Präteritum alemán). Este concepto representa el terminus ad quem del proceso que debe guiar a su modificación o "variación" (Variation). Y al mismo tiempo, es también el terminus a quo de otro proceso que debe permitir su "restitución" (Restitution) final (cf. Sommer 1985, 62). En otras palabras, el filósofo empiriocriticista no tiene sólo la tarea limitada de describir el concepto natural de mundo tal cual fue antes de que se presentara cualquier tipo de actitud teorética, sino que es más importante para él la "restitución" de ese concepto original después de su modificación, la cual se da en consecuencia del proceso que Avenarius llama "introyección" (Introjektion). Sin embargo, antes de dar cuenta de la noción de introyección, primero debemos llevar a cabo la descripción del contenido del concepto natural de mundo. La cuestión que debemos analizar es la siguiente: ¿cuáles son las estructuras peculiares del mundo en un principio? Concretamente antes de cualquier modificación o alteración, y ésta encuentra una respuesta en los dos principios de Avenarius: la coordinación principal empiriocriticista y la hipótesis fundamental de la equivalencia humana.

\section{§ 5. La "CoORdinación Principal" y la "Hipótesis Fundamental de la Equivalencia HUMANA" EMPIRIOCRITICISTA}

El primero de los elementos que caracterizan nuestro acceso primario y natural al mundo es expresado por Avenarius en la fórmula empiriocriticista siguiente: el "Coordinación principal (Prinzipialkoordination)". Éste es un término

\footnotetext{
- "Ich mit all meinen Gedanken und Gefühlen fand mich inmitten einer Umgebung. Diese Umgebung war aus mannigfaltigen Bestandteilen zusammengesetzt, welche untereinander in mannigfaltigen Verhältnissen der Abhängigkeit standen. Der Umgebung gehörten auch Mitmenschen an mit mannigfaltigen Aussagen; und was sie sagten, stand zumeist wieder in einem Abhängigkeitsverhältnis zur Umgebung. [...] Alles, wie ich selbst auch: und so dachte ich nicht anders, als dass Mitmenschen Wesen seien wie ich ich selbst Wesen wie sie. Das war die Welt, wie ich sie am Anfang meines Philosophierens als ein Seiendes, Sicheres, Bekanntes, Vertrautes, Begriffenes vorfand".
} 
técnico que da nombre a la " Copertenencia e inseparabilidad de la experiencia del yo con la experiencia del entorno en toda experiencia que se realiza"7 (Avenarius $1891, \mathrm{Nr} .148,83)$. Este principio empiriocriticista, tal como lo ha señalado Moritz Schlick, puede ser visto nada más y nada menos que como una reformulación de la famosa afirmación schopenhaueriana: no hay objeto sin sujeto (cf. Schlick 1925, 201 ss.; Lenin 1972, 67 ss.). Esto significa que ningún elemento o parte del "medio circundante" (Umgebung) es posible sin alguna cierta correlación con un sujeto. Aunque también el reverso puede ser el caso: un sujeto no es posible sin tener alguna relación con algún objeto. Este es un principio que se sigue directamente de la estructura esencial del medio circundante como tal. En cada medio circundante, el sujeto o "Yo-designado (IchBezeichnete)", como Avenarius frecuentemente lo denomina, es un "miembro central" (Zentralglied) en relación con el cual están siempre estructuradas las partes componentes del medio circundante en una 'coordinación principal' como 'miembros opuestos' (Gegenglieder). La hipótesis de un sujeto sin mundo así como la de un mundo sin sujeto se origina sólo como una consecuencia de la variación del concepto natural de mundo. Es producto de un pensamiento teorético, no de una descripción de lo que es dado originariamente (cf. Bermes 2004, 120).

Algo que es igualmente esencial y complementario a la coordinación principal del empiriocriticismo es, de acuerdo con Avenarius, la "hipótesis fundamental de la equivalencia humana" (Grundannahme der menschlichen Gleichheit). Lo que encontramos en el concepto natural de mundo no son sólo las cosas físicas, como los árboles, los perros y las montañas, tampoco encontramos sólo nuestros estados mentales; sino que un componente fundamental de mi medio circundante está representado por mis "prójimos" (Mitmenschen). Ellos son percibidos por mí no sólo como miembros opuestos de mi coordinación principal, y por tanto, como componentes de mi medio circundante, sino también como componentes centrales de otra coordinación principal, la suya, y que además no coincide con la mía. A pesar de ello, es importante notar que esta descripción no representa para Avenarius un "hallazgo" empiriocriticista, es decir, que no es el resultado de una mera observación y de una descripción del

\footnotetext{
7 "Zusammengehörigkeit und Unzertrennlichkeit der Ich-Erfahrung mit der Umgebungserfahrung in jeder Erfahrung, welche sich verwirklicht". 
concepto natural de mundo. Por el contrario, la "hipótesis fundamental de la equivalencia humana" es por definición una hipótesis y, más precisamente, el resultado de una interpretación no-mecanicista de los movimientos y de los ruidos producidos por mis prójimos como si fueran componentes de mi medio circundante (cf. Avenarius 1891, Nr. 14, 9). El carácter interpretativo de la "equivalencia humana" es algo completamente necesario, porque si mis prójimos representaran un papel principal en la coordinación principal, con los mismos derechos que yo, entonces la propia estructura del medio circundante colapsaría finalmente. De hecho, un medio circundante siempre implica una relación entre un miembro singular central y una pluralidad de miembros opuestos. $Y$ esa pluralidad de miembros centrales no puede ser dada dentro de un único medio circundante; por otra parte, una pluralidad de medios circundantes, cada una con su propio miembro central, es lógicamente posible y encuentra expresión en la hipótesis fundamental de la equivalencia humana que es sostenida por el empiriocriticismo.

\section{$\S$ 6. La Disputa sobre el Ego del Concepto natural de Mundo}

En este momento es necesario introducir una clarificación de suma importancia. Como ha señalado apropiadamente Christian Bermes, "Lo que Avenarius presenta aquí con la expresión «concepto inicial de mundo», de ninguna manera puede ni debe ser identificado con una reducción de un supuesto «mundo» independiente de sujetos a un 'medio circundante' subjetivo" (Bermes 2004, 118). El motivo de este señalamiento es que de acuerdo con Avenarius el concepto natural de mundo no entraña una diferenciación esencial entre los modos de darse, por una parte del Ego y por otra de los otros componentes del medio entorno (Umgebung). Por eso, Avenarius prefiere usar la expresión "Yodesignado (Ich-Bezeichnete)" cuando se refiere al miembro central de una coordinación principal, en vez de solamente llamarlo "Yo (Ich)": este último término sugeriría una posición substancialista respecto de la naturaleza del Ego. Por eso Avenarius afirma lo siguiente: "El yo y el entorno [...] están, respecto de su estar dado, perfectamente en la misma línea" ${ }^{8}$ (Avenarius 1891, Nr. 143, 82). En ese caso, la percepción de un árbol tiene el mismo estatus y comparte el mismo modo de darse de la percepción del Ego. La diferencia entre ambas

\footnotetext{
8 "Ich und die Umgebung [ ..] stehen hinsichtlich inres Gegebenseins vollständig auf gleicher Linie".
} 
reside solamente, y por decirlo así, en la 'intensidad' de ambas percepciones. " El yo difiere de los elementos de su entorno a través de una riqueza y multiplicidad más grandes [...]; pero no se diferencía por el modo general como son experienciados los elementos del yo y los del entorno" ${ }^{\prime 9}$ (Avenarius 1891, Nr. 145, 82 s.).

Esta característica que se da en la explicación de Avenarius sobre el concepto natural de mundo llegó a ser el núcleo esencial de la disputa que tuvo con sus contemporáneos. El filósofo alemán Wilhelm Schuppe, de a cuerdo a Jospeh Petzoldt, quien a su vez es uno de los fundadores del Empirocriticismo junto con Mach y Avenarius, se enfrenta en una carta pública, escrita en 1893 y publicada después como un apéndice a la cuarta edición de El concepto humano de mundo a esto último, es decir, al hecho de que el concepto de mundo natural está esencialmente basado en un sujeto que percibe su medio circundante y que hace deducciones a partir de lo que es dado originariamente. En este sentido, su idea es que tal vez Avenarius debería haber incluido en su descripción de la experiencia natural del mundo también la experiencia de un Ego, en tanto que ésta es un componente necesario y no reducible a la experiencia de los otros miembros del medio entorno. Sin embargo, Avenarius contesta de la siguiente manera a la interpretación de Schuppe: "A la pregunta: "qué es el yo?" Sería yo capaz de responder también hoy solamente: El "yo" es un sustituto lingüístico de un gesto indicador -una palabra"10 (Avenarius 1927, 175). Nuevamente Avenarius rechaza la absolutización del Ego, lo cual significaría, a mi juicio, una anulación del principio de coordinación así como una corrupción substancialista de los datos de la experiencia pura. Es fundamental tener en mente este elemento de la doctrina de Avenarius sobre el concepto natural de mundo, porque, como veremos enseguida, constituye una de las diferencias fundamentales entre el Empirocriticismo y la aproximación fenomenológica de Husserl.

\section{$\S$ 7. El Empleo husserliano del Concepto natural de Mundo}

EN SUS LECTURAS DE GOTINGA

\footnotetext{
9 "Das Ich differiert von den Bestandteilen seiner Umgebung wohl durch eine größere Reichhaltigkeit und Mannigfaltigkeit [...]; aber es unterscheidet sich nicht durch die allgemeine Art wie die Bestandteile des Ich und diejenigen der Umgebung erfahren werden".

10 "Auf die Frage: 'Was ist das Ich?' vermöchte ich auch heute nur zu antworten: Das 'Ich' ist ein sprachlicher Ersatz einer hinzeigenden Geste - ein Wort".
} 
Hasta ahora hemos tratado de exponer la explicación de Avenarius sobre el concepto natural de mundo, aunque también es cierto que no hemos podido dar cuenta de todos sus detalles. Más bien hemos tenido que hacer una selección de temas importantes para el argumento principal de este artículo, en el cual queremos analizar la relación entre las filosofías de Husserl y Avenarius, de manera principal en lo que concierne al tema del concepto natural de mundo.

Especialmente después de la publicación del volumen XXXIX de Husserliana, intitulado El mundo de la vida. Interpretaciones del mundo predado y su constitución (Die Lebenswelt. Auslegungen der vorgegebenen Welt und ihrer Konstitution), se ha vuelto lugar común en la literatura secundaria constatar el hecho de que las numerosas descripciones husserlianas de la actitud natural pueden rastrearse en las primeras descripciones de Avenarius del concepto natural de mundo (cf. Sommer 1984; Sowa 2008; Soldinger 2010; Moran 2013). Y esta afirmación tiene buenas razones para ser sostenida. Es suficiente con echarle un vistazo a la primera introducción que Husserl hace del concepto natural de mundo en las Lecciones de 1907, comúnmente llamadas Dingkolleg ${ }^{11}$, para dar cuenta de la similitud entre ambas posiciones. En la prima parte de estas lecturas, intitulada Die Idee der Phänomenologie, Husserl habla de la "actitud natural del espíritu o del pensamiento (natürliche Denk- bzw. Giesteshaltung)" para caracterizar una orientación directa hacia las cosas y la contrasta con una "actitud filosófica del espíritu (philosophische Geisteshaltung)", que es capaz de reflexionar e interrogar por la relación entre el sujeto y el objeto del conocimiento, y por lo cual es la vía de acceso para cualquier posibilidad de la teoría del conocimiento (cf. Hua II, § 1). Sin embargo, es en la segunda sección de Dingkolleg donde Husserl expone su noción de "natürliche Geisteshaltung", con una referencia implícita a la posición de Avenarius sobre el concepto natural de mundo En esta sección Husserl escribe lo siguiente: "En la actitud espiritual natural hay para nosotros un mundo existente ante los ojos, un mundo que se extiende sin fin en el espacio, que es ahora, ha sido antes y será en el futuro, y que consiste en una inagotable plenitud de cosas, que ya duran, ya se transforman, se entrelazan entre si y vuelven a separarse, ejercen efectos unas sobre otras y los padecen unas de otras. En este mundo nos integramos noso-

\footnotetext{
${ }^{11}$ Nota del traductor. Se trata de una serie de lecciones impartidas por Husserl en 1907 en Gotinga, actualmente publicadas en la edición de sus obras completas (Husserliana), tomos II y XVI.
} 
tros mismos, como a él, nos encontramos nosotros también a nosotros mismos, y nos encontramos en medio de este mundo. Una posición destacada nos es propia en él: nos encontramos a nosotros como un centro de relaciones con el resto del mundo en cuanto nuestro medio circundante" ${ }^{12}$ (Hua XVI, 4). Lo que es digno de atención es la forma en que se caracteriza a la relación intramundana entre el sujeto y el mundo, en tanto que es una relación entre un "centro de relaciones (Beziehungszentrum)" y el relativo "medio circundante (Umgebung)", que claramente esconde el concepto de Avenarius y del empiriocriticismo que hemos expuesto anteriormente, es decir, el principio de coordinación.

De manera similar, Husserl introduce sus reflexiones concernientes a este tema en las las lecturas del curso de Invierno de 1910/11, que anteriormente hemos mencionado, con una alusión indubitable al método empiriocriticsta. "Antes de toda teoría", afirma Husserl, " el mundo está dado. Todas las opiniones, correctas o incorrectas, populares, supersticiosas, científicas, todas se refieren al mundo ya predado"13 (Hua XIII, Beilage XXII, 196). Sin embargo, el mundo es caracterizado aquí por Husserl todavía como el 'hallazgo' primario (Vorgefunden) en relación con una "actitud" específica. La introducción del concepto natural de mundo está entonces directamente asociada con una discusión sobre los diversos tipos de actitudes; concretamente de los actos en el que las entidades les son dadas al sujeto y de acuerdo a su modo particular de donación. De hecho, la lectura inicia "con una descripción de las diferentes actitudes, en las que experiencia y conocimiento pueden tener lugar, primero de la actitud natural, en la cual todos nosotros vivimos y de la cual por lo tanto partimos cuando realizamos la modificación filosófica de la mirada"14 (Hua XIII, Nr. 6, 112). La subsecuente descripción de la actitud natural, que Husserl comienza en los $\S \S 1-5$, contiene un tipo de resumen de los temas que ocupan la parte

\footnotetext{
12 "In der natürlichen Geistshaltung steht uns seine seiende Welt vor Augen, eine Welt, die sich endlos im Raum ausbreitet, jetzt ist und vorher gewesen ist und künftig sein wird; sie besteht aus einer unerschöpflichen Fülle von Dingen, die bald dauern und bald sich veränder, sich miteinander verknüpfen und sich wieder trennen, aufeinander Wirkungen üben und solche voneinander leiden. In dieser Welt ordnen wir uns selbst ein, wie sie finden wir uns selbst vor, und finden uns inmitten dieser Welt vor. Eine ausgezeichnete Stellung eignet uns in dieser Welt: Wir finden uns vor als ein Beziehungszentrum zu den übrigen Welt als unserer Umgebung".

13 "Vor aller Theorie ist die Welt gegeben. Alle Meinungen, rechtmäßige oder unrechtmäßige, populäre, abergläubliche, wissenschaftliche, alle beziehen sich auf die schon vorgegebene Welt".

14 "Mit einer Beschreibung der verschiedenen Einstellungen, in denen Erfahrung und Erkenntnis statthaben kann, zunächst der natürlichen Einstellung, in der wir alle leben und von der wir also ausgehen, wenn wir die philosophische Blickänderung vollziehen".
} 
central de sus lecturas en Gotinga en la primera década del siglo XX. Los problemas más importantes son específicamente los siguientes: el cuerpo y su entorno espacio-temporal, la localización de las sensaciones en el cuerpo, el problema de la Einfühlung, la constitución del espacio objetivo euclidiano y el problema correlativo de la normatividad en la percepción. La riqueza se las descripciones fenomenológicas expuestas por Husserl tal vez tengan poco que ver con el vocabulario verdaderamente abstracto del empiriocriticismo, pero es ciertamente posible que Husserl haya utilizado las formulaciones de Avenarius como un esbozo, como un esquema para organizar el material de sus investigaciones y alcanzar así una exposición coherente de lo que es dado en la actitud natural y pre-filosófica.

\section{§ 8. La "ReVersión" (UMKEHRunG) hUSSerLiana de LA Doctrina de Avenarius}

Un descubrimiento crucial de la discusión previa está representado seguramente por el descubrimiento de ciertas afinidades innegables entre la concepción de Avenarius sobre el concepto natural de mundo y la aplicación que Husserl hace de él en sus descripciones del mundo natural, en tanto que éste sería el correlato de la actitud natural original y pre-filosófica en sus lecturas de Gotinga. Lo que resta por hacer, para tener una perspectiva adecuada de la relación entre ambos filósofos, es determinar la propia valoración que hace Husserl del Empiriocriticismo de Avenarius.

En el manuscrito asociado con algunas lecturas del curso Grundprobleme der Phänomenologie y en el cual es más preponderante la influencia de la noción de Avenarius del concepto natural de mundo, mucho más que en algunos otros escritos, encontramos la siguiente afirmación, que a su vez es lacónica: "El comienzo es bueno en Avenarius, pero se queda atascado"15 (Hua XIII, Beilage XXII, 199). ¿Qué quiere decir con esto? Esta afirmación puede tener diferentes significados, especialmente dependiendo de cómo interpretemos las filosofías de Husserl y de Avenarius. En esta sección final voy a proponer mi interpretación.

El inicio de Avenarius es "bueno (gut)", y su filosofía representa "una tendencia muy valiosa (eine sehr wertvolle Tendenz)" como Husserl mismo admite

15 "Der Anfang ist bei Avenarius gut, aber er bleibt stecken". 
en este texto (Hua XIII, Beilage XXII, 197), esencialmente porque una descripción del mundo natural, esto es del mundo tal cual aparece primariamente para el observador que vive a través de la actitud natural, es considerada como algo absolutamente fundamental e inevitable en los primeros pasos para ganar un conocimiento verdadero y absoluto. El principio de la ausencia de cualquier tipo de presuposición (Voraussetzungslosikeit), y que tanto Husserl como Avenarius tienen en común, requiere una primera tematización del mundo tal cual es dado antes y después de las caracterizaciones teoréticas, lo que significa, antes y después de las teorías científicas y filosóficas. Avenarius juzga que ésta es la tarea principal de su filosofía, o todavía más lejos, de la filosofía misma, por tanto, siempre está presente la necesidad de describir las bases naturales que yacen bajo el conocimiento teorético. Concretamente podríamos decir que él dedicó su vida intelectual al esfuerzo de dar cuenta fidedignamente de las estructuras de la actitud natural y pre-teorética. El descubrimiento de la donación natural es tal vez una de las más grandes deudas que Husserl reconoce tener con la filosofía de Avenarius. En un manuscrito todavía no publicado y que probablemente data de 1926, Husserl afirma lo siguiente en relación con su teoría del mundo de la vida (Lebenswelt): "Mi planteamiento originario [fue] animado por la doctrina positivista del concepto natural de mundo de Avenarius: la descripción científica del mundo puramente como mundo de la experiencia."16 (Ms. A VII 20 / 471). Sin embargo, además de este reconocimiento, Husserl también da cuenta de que algo fue omitido en la concepción de Avenarius, y esa omisión es algo que motivó, o mejor dicho, que obligó al filósofo a brincarse la actitud natural y su correlato, el mundo natural.

En un apéndice del curso de verano de 1925, en las lecturas Phänomenologische Psychologie, Husserl nota que es necesaria una " reversión total de la doctrina de Avenarius (totale Umkehrung der Lehre von Avenarius)" en vista de que es verdad que "ninguna filosofía transcendental puede renunciar al ser del mundo experiencial, pero que bien puede hacerlo trascendentalmente comprensible"$^{17}$ (Hua IX, Beilage XXII, 474). Lo que le hace falta a la descripción del concepto natural de mundo de Avenarius es precisamente este carácter

\footnotetext{
16 "Meine ursprüngliche Fragestellung [war] angeregt durch Avenarius' positivistische Lehre vom natürlichen Weltbegriff: die wissenschaftliche Deskription der Welt rein als Welt der Erfahrung".

17 "Keine Tranzendentalphilosophie das Sein der Erfahrungswelt preisgeben kann, aber wohl, dass sie sie transzendental verständlich machen kann".
} 
trascendental. Avenarius reconoció que es imposible para la filosofía olvidarse del ser del mundo experiencial, pero él renunció a hacerlo "trascendentalmente comprensible". En otras palabras, la insuficiencia del enfoque de Avenarius consiste en no haber valorado lo que Husserl ha denominado la "paradoja de la subjetividad trascendental".

\section{§ 9. Conclusión. La Reducción Fenomenológica como la Apertura Metódica de la "Paradoja de la Subjetividad"}

En un famoso parágrafo de su último trabajo, La Crisis de las Ciencias Europeas y la Fenomenología Trascendental (1936), Husserl sostiene que Kant y las filosofías trascendentales del pasado no pudieron dar cuenta por completo de la paradoja de la subjetividad humana (cf. Carr 1999). Esta paradoja consiste en el hecho de que " nosotros somos en él [el mundo de la vida] objeto entre objetos $[\ldots]$; a saber, como existentes aquí y allá, en certeza experiencial simple, antes de toda cuestión científica, sea fisiológica, psicológica, sociológica etc. Por otro lado, somos sujetos para este mundo, es decir, sujetos-yo que lo experimentan, que lo piensan, que están referidos a él en la práctica, para los cuales este mundo-entorno (Umwelt) tiene sólo el sentido de ser que nuestras experiencias, nuestros pensamientos, nuestras valoraciones etc. respectivamente le han dado"18 (Hua VI, § 53, 107; cf. Hua III/1, 79 s., Hua XXVII, 138, 157, Hua I, 128). A la luz de esta descripción de la paradoja, debemos decir que el enfoque de Avenarius del concepto natural de mundo también sufre de esta misma carencia, porque no logró asir la paradoja de la subjetividad.

Una evidencia contundente está representada en la disputa sobre el Ego que se da en su teoría del concepto natural de mundo y que hemos esbozado anteriormente. Como hemos intentado enfatizar, la tesis de Avenarius es que el Ego no tiene prioridad epistemológica, o si ponemos el dedo en la llaga, vemos que el Ego tampoco tiene prioridad trascendental si lo comparamos con cualquier otro objeto del medio entorno. A pesar de ello, este rechazo del estatus

\footnotetext{
18 "Wir sind in ihr [die Lebenswelt] Objekte unter Objketen [...]; nämlich als da und dort seiende, in schlichter Erfahrungsgewißheit, vor allen wissenschaftlichen, sei es physiologischen, psychologischen, soziologischen usw. Feststellungen. Wir sind andererseits Subjekte für diese Welt, nämlich als die sie erfahrenden, bedenkenden, zwecktätig auf sie bezogenen Ichsubjekte, für welche diese Umwelt nur den Seinsinn hat, den ihr unsere Erfahrungen, unsere Gedanken, unsere Wertungen usw. jeweilig gegeben haben".
} 
trascendental del sujeto -que en Avenarius tiene ciertamente un sabor antikantiano- lo lleva a lo que Husserl llama, en la tercera sección de Ideas II, al "olvido del espíritu". Esto significa que el espíritu, en este caso la subjetividad trascendental, ya no es capaz de reconocer en el mundo natural ningún producto de sus propios logros constitutivos. Este es el olvido que caracteriza a la propia actitud natural, para la cual el mundo es primero y antes que nada algo 'dado' y no un 'producto' de constitución trascendental.

Dadas estas premisas, podemos ahora dar nueva luz a la presentación de la actitud natural en los $\S \S 27-30$ de Ideas I y consecuentemente introducirnos en el método fundamental de Husserl, que no es sino la reducción fenomenológicotrascendental. La filosofía no puede detener su reflexión en el nivel de la actitud natural, como en el caso de Avenarius. La descripción del concepto natural de mundo no es per se suficiente para revelar la verdad absoluta del idealismo trascendental, a saber, el hecho de que el mundo existe sólo para la conciencia, y de que hablar de un mundo sin ninguna relación con una conciencia absoluta conlleva a un sinsentido tan grande como hablar de un círculo cuadrado (cf. Hua III/1, § 55, 120). Por esta razón, Husserl necesita en la "Consideración fundamental (Fundamentalbetrachtung)" de las Ideas I introducir primero la "puesta entre paréntesis (Einklammerung)" del mundo natural y la "desconexión (Ausshaltung)" de su tesis general y, sucesivamente, introducir el método de la reducción de las verdades naturales empíricas hacia lo trascendental y sus verdades fenomenológicas. El método de la reducción es lo que hace falta en la concepción de Avenarius sobre el mundo natural y lo que le habría permitido descubrir la paradoja de la subjetividad trascendental. 


\section{BIBLIOGRAFIA}

Avenarius, Richard Heinrich Ludwig (1868): Über die beiden ersten Phasen des Spinozistischen Pantheismus und das Verhältnis der zweiten zur dritten Phase. Nebst einem Anhang: Über Reihenfolge und Abfassungszeit der älteren Schriften Spinozas. Leipzig: Eduard Avenarius.

- (1876): Philosophie als Denken der Welt gemäß dem Prinzip des Kleinsten Kraftmaßes. Prolegomena zu einer Kritik der reinen Erfahrung. Leipzig: Fues.

- (1888): Kritik der reinen Erfahrung. Leipzig: Fues.

- (1891): Der menschliche Weltbegriff. Leipzig: O.R. Reisland.

- (1927): Der menschliche Weltbegriff. 4., unveränderte Auflage, wie die dritte vermehrt um den Abdruck von Wilhelm Schuppe, Offener Brief an Avenarius über die "Bestätigung des naiven Realismus" und von Richard Avenarius "Bemerkungen zum Begriff des Gegenstandes der Psychologie". Leipzig: O. R. Reisland.

Bermes, Christian (2004): Welt als Thema der Philosophie. Vom metaphysischen zum natürlichen Weltbegriff. Phänomenologische Forschungen, Beiheft 1. Hamburg: Meiner Verlag.

CaIRns, Dorion (1976): Conversations with Husserl and Fink. Phaenomenologica, vol. 55. Den Haag: Martinus Nijhoff.

CARR, David (1999): The Paradox of Subjectivity. The Self in the Transcendental Tradition. Oxford: Oxford University Press.

Embree, Lester, and Thomas Nenon (eds.). (2013): Husserl's Ideen. Contributions to Phenomenology, vol. 66. Dordrecht: Springer.

Heidegger, Martin. (1979): Sein und Zeit. Tübingen: Max Niemeyer.

- (2009) Ser y Tiempo. Madrid: Trotta.

HUSSERL, Edmund (1952): Ideen zu einer reinen Phänomenologie und phänomenologischen Philosophie. Zweites Buch: Phänomenologische Untersuchungen zur Konstitution. Husserliana IV. Den Haag: Martinus Nijhoff.

- (1962): Die Krisis der europäischen Wissenschaft und die transzendentale Phänomenologie. Eine Einleitung in die phänomenologische Philosophie. Husserliana VI. Den Haag: Martinus Nijhoff.

- (1976): Ideen zu einer reinen Phänomenologie und phänomenologischen Philosophie. Erstes Buch: Allgemine Einführung in die reine Phänomenologie. Husserliana III/1. Den Haag: Martinus Nijhoff.

- (2006): Investigaciones Lógicas, 1. Versión de Manuel G. Morente y José Gaos. Madrid: Alianza Editorial.

- (2013); Ideas relativas a una fenomenologia pura y una filosofía fenomenológica. Libro Primero: Introducción general a la fenomenologia pura. Nueva edición y refundición integral de la traducción de José Gaos por Antonio Zirión Quijano. México: Fondo de Cultura Económica.

LAVIGNE, Jean-Francois (2003): "Husserl lecteur d'Avenarius: une contribution à la genèse de la réduction phénoménologique?". En: KAIROS (22): 61-82.

LENIN, Vladimir I. (1972): Materialism and Empirio-Criticism. Peking: Foreign Languages Press.

MORAN, Dermot (2013): "From the Natural Attitude to the Life-World". En: Husserl's Ideen, ed. Lester Embree y Thomas Nenon. Contributions to Phenomenology, vol. 66. Dordrecht: Springer, 105-124.

SCANLON, John (1988): "Husserl's Ideas and the Natural Concept of the World". En: Edmund Husserl and the Phenomenological Tradition, ed. Robert Sokolowski. Studies in Philoso- 
phy and the History of Philosophy, vol. 18. Washington, DC: Catholic University of America Press, 217-233.

- (1996): "Objectivity and Introjection in Ideas II". En: Issues in Husserl's Ideas II, ed. Thomas Nenon and Lester Embree. Contributions to Phenomenology, vol. 24. Dordrecht: Kluwer Academic Publishers, 213-222.

SCHLICK, Moritz (1925): Allgemeine Erkenntnislehre, 2nd ed. Berlin: Julius Springer.

SOldinger, Emanuele (2010): "Husserls Auseinandersetzung mit Avenarius und Mach und ihr Verhältnis zur Lebensweltproblematik". En: Geist - Person - Gemeinschaft: Freiburger Beiträge zur Aktualität Husserls, ed. Philippe Merz, Andrea Staiti and Frank Steffen. Würzburg: Ergon Verlag, 189-217.

SOMmER, Manfred (1984): "Einleitung: Husserls Göttinger Lebenswelt". En: Die Konstitution der geistigen Welt, ed. Manfred Sommer. Philosophische Bibliothek, vol. 369. Hamburg, IX-XLII.

- (1985): Husserl und der frühe Positivismus. Frankfurt am Main: Vittorio Klostermann.

SowA, Rochus (2008): "Einleitung des Herausgebers". En: Husserl, Edmund: Die LebensweltAuslegungen der vorgegebenen Welt und ihrer Konstitution. Dordrecht: Kluwer. 